\title{
THE TEST FIELD FOR UAV ACCURACY ASSESSMENTS
}

\author{
Paweł Wiącek, Krystian Pyka \\ AGH University of Science and Technology \\ paw.wiacek@gmail.com, krisfoto@agh.edu.pl
}

Commission I, WG I/10

KEY WORDS: UAV, PPK, bundle adjustment, mapping, direct georeference

\begin{abstract}
:
Nowadays UAV photogrammetry becomes a common method for mapping and surveying. At the same time due to the increasing range of work carried out with UAV, the importance of final product accuracy increases. However to obtain survey-grade accuracy it is necessary to perform bundle adjustment processes that could be affected by multiple factors like unstable camera calibration, correlation between interior and exterior orientation and insufficient georeference information. One of the aims of the project was to prepare the terrestrial test field, which helps to obtain optimal decorrelation and allows to objectively assess the accuracy of the bundle adjustment in UAV application. During the project, two multi-variant flights over the test field were conducted. The flights were performed with a fixed-wing airframe equipped with PPK receiver on-board. Based on the conducted flights, many data sets have been prepared, which differ as follows: types of cameras, GSD, flight direction and georeferenced method.
\end{abstract}

\section{INTORODUCTION}

The use of drones in surveying is developing rapidly and areas of UAVs application are getting the more and more various (Nex, 2014). Some types of surveys, such as natural hazards inventorying, vegetation monitoring, cultural heritages mapping and many others, need more good interpretation condition, then very high geometrical trueness and precision. At the same time, there are surveys such as cadastral mapping or base map elaboration which require accuracy on a few centimetre level (Kurczynski, Bakuła, 2016; Kędzierski, Fryskowska, 2016; van Hinsberg, Cramer, 2013).

Among many publications on UAV-based aerial surveying, relatively little of them are concerned with matters of factors negatively affecting the accuracy. The problem is not trivial due to the fact, that the fundamental condition for achieving a correct rays intersection is the knowledge of external and internal cameras orientation parameters. Unfortunately, the estimation of those parameters is affected due to their high correlation. On this account the classical photogrammetry capture images using metric cameras which internal is orientation is performed autonomously in laboratory or in field test. This way does not work in UAV survey application due to using light consumer-grade cameras with unstable internal orientation. In practice, the only solution for UAV photogrammetry is performing the self-calibration (in-situ) as an integral part of camera pose estimation using structure-frommotion approach. Admittedly the self-calibration bundled in SFM seems as simple process, but there is a question about actual accuracy and reliability (Luhman, 2015). The result of self-calibration is very sensitive to structure of image block and morphology of mapped area.

The issue of poor stability of internal orientation of cameras was described by Cramer and co-authors (Cramer, 2017). The research has showed out that in some cameras the focal length and principal point position are changing in several dozen of microns, although between calibration the camera was not operationally used. It is worth underlining that also thermal conditions impact the parameters of interior orientation (Daakir, 2019).

The standard method of image orientation, called indirect georeferencing, is a rigorous solution by bundle adjustment using Ground Control Points. The need to measure GCPs significantly increases the work time and the cost of UAVbased mapping. The fundamental question is about the minimal number of GCPs and its localization in elaborated area which are need to achieve a good precision of image orientation. Most papers express the view that the GCPs number should be between 3 and dozen, and suggest 6 GCPs as an optimal number (James, 2017). Several authors advise more than 2 GCPs per 100 photos (Sanz-Ablanedo, 2018). Due to various characteristic of mapped area (size, shape, relief, land cover) there are no universal rules about optimal number of GCPs and their spatial distribution.

The direct georefeencing of UAV images has been developed for some years. Nowadays, many vendors install survey-grade RTK-GNSS receiver on board the UAV. The application of cameras pose with centimetre accuracy in SFM workflow opens the door to UAV elaboration without GCPs. There are some experiences that confirm the expectation (Gerke, Stöcker, 2019).

The main aim of the research was to investigate the influence of the georeferencing method and camera calibration stability on the final accuracy.

Based on the conducted flights, many data sets have been prepared, which differ as follows:

- $\quad$ flight parameters: (1) GSD 2 and $3 \mathrm{~cm}$, (2) direction NS and W-E, (3) overlap 60/60 and 70/70

- cameras: (1) with ant (2) without rolling shutter

- $\quad$ georeferencing method: (1) only GCP, (2) only direct georeference (PPK), (3) combined

-

In all test, about 150 Control Points were used to evaluate the final accuracy. 


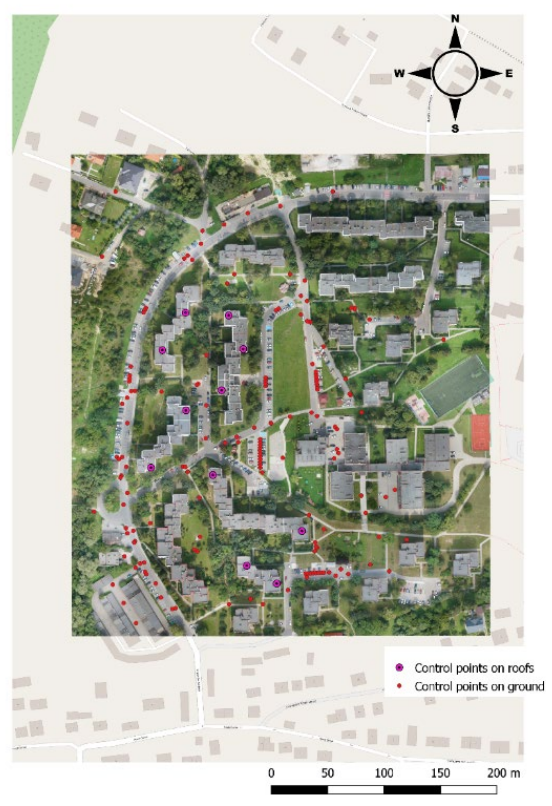

a) Roof Control Points and Check Points

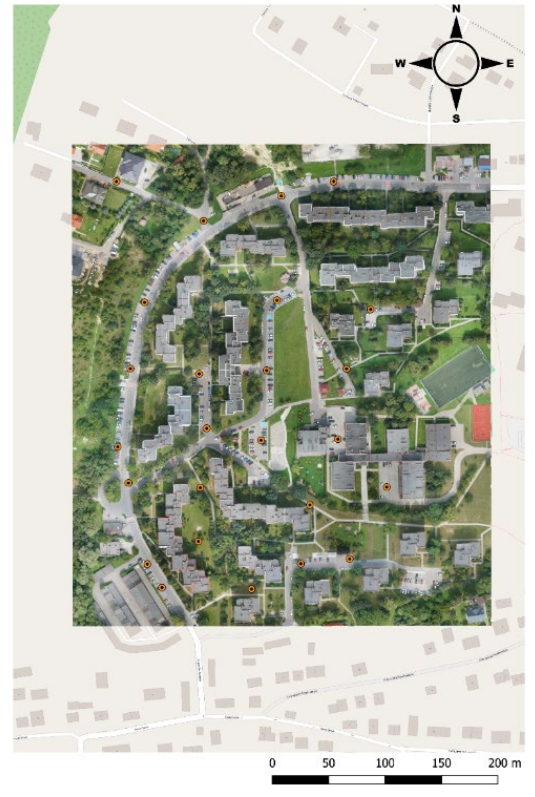

b) Ground Control Points

Figure 1 Test field overview

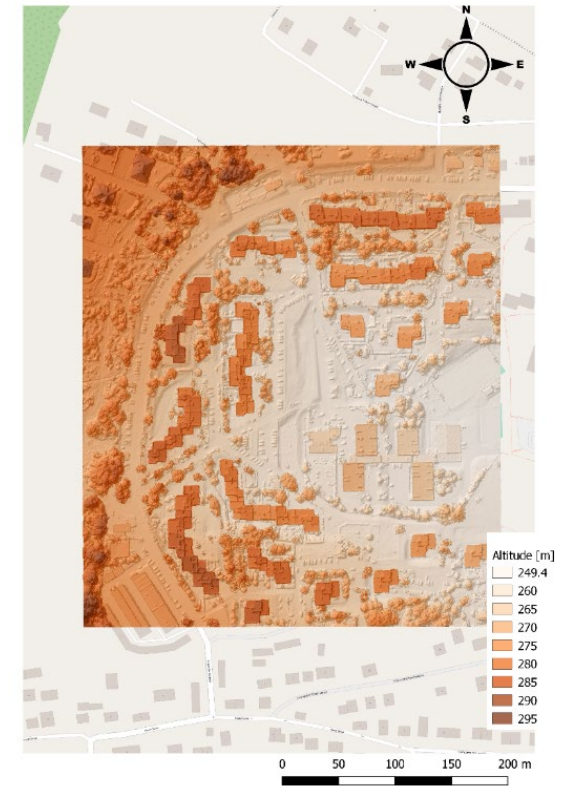

c) Test field area altitude

\section{TEST FIELD}

Firstly, to investigate the accuracy of UAV-based aerial photogrammetry and factors affecting it, special test field was prepared. It was located in residential area in Bochnia city covered area of average $350 \mathrm{~m} \times 400 \mathrm{~m}$. The size of the test field has been chosen so as to enable multi-variant coverage within one flight with fixed wing UAVs or full coverage in one flight with multirotor UAVs. The area of the test field is characterized by a large number of internal roads, several storey blocks and terrain altitude differences up to 35 meters. Thanks to this, it was possible to evenly distribute about 150 control/check points across the entire area (Figure 1).

The points were divided into three groups:

I. Ground Control Points - 25 natural marked points used during bundle adjustment process

II. Roof Check Points - 11 marked points located on building roofs

III. Check Points - about 150 natural marked points used for accuracy determination measured over final products in GIS software

As Ground Control Points and Check Points natural points like: parking lines and wells were adopted while as Roof Check Points special targets made of PVC were used (Figure 2). Control points were measured in three session from June 2018 to December 2018 using RTK and RTN method Based on these measurements; the accuracy of the points on $2 \mathrm{~cm}$ horizontal and $3 \mathrm{~cm}$ vertical level was determined(Table 1).

\begin{tabular}{|l|c|c|c|}
\hline & $\mathrm{X}$ & $\mathrm{Y}$ & $\mathrm{Z}$ \\
\hline Mean difference & -0.005 & 0.007 & 0.015 \\
\hline Stand. deviation & 0.018 & 0.021 & 0.024 \\
\hline RMSE & 0.018 & 0.022 & 0.028 \\
\hline
\end{tabular}

Table 1 Assessment of check points measurements accuracy
Two multi-variants flights were conducted using FlyTech UAV BIRDIE fixed wing equipped with different cameras for each flight. (Figure 3). Each multivariant flight consisted of five missions varying in GSD/flight altitude, overlap and flight direction. What is important, between each configuration there was no middle landing, which was possible thanks to advanced mission planning options in Mission Manager software.

The first flight was made with Sony RX1R II camera at respectively $155 \mathrm{~m}$ and $230 \mathrm{~m}$ above mean ground level. The second flight was made with Sony a6000 camera with Voigtlander Color Skopar $21 \mathrm{~mm}$ lens at respectively $110 \mathrm{~m}$ and $160 \mathrm{~m}$ above mean ground level. Both flight were made in one day with the same weather conditions.

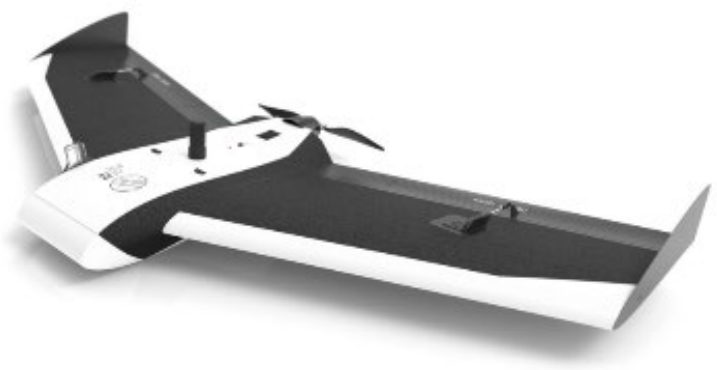

Figure 3 FLY TECH UAV - BIRDIE

Additionally for further calculation, due to expected positive effect on the in-situ calibration of the cameras, flight variants were extended by three image sets consisting of two cross flight directions (variants from 6 to 8 ). 
a)

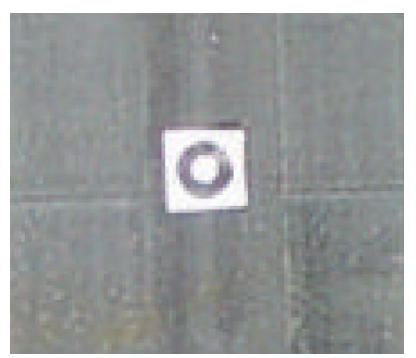

b)

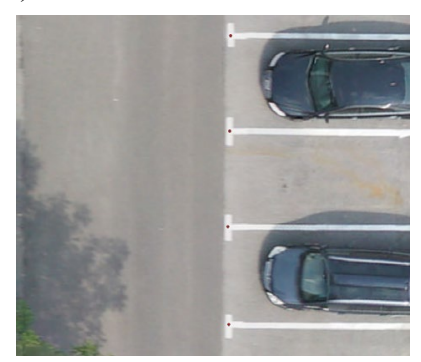

c)

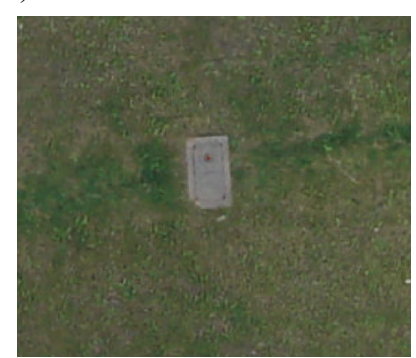

d)

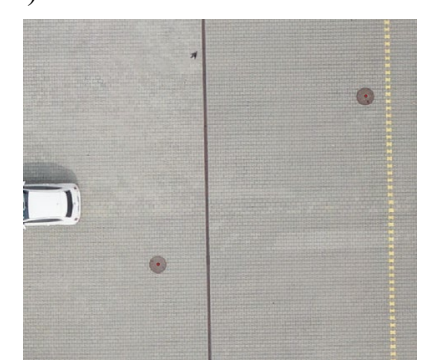

Figure 2. a) roof points, b) ground points - parking lines, c) ground points - telecommunications well,

d) ground points - sewage well

\begin{tabular}{|l|l|l|}
\hline Model & Sony a6000 & Sony RX1R II \\
\hline Image Sensor & APS-C $(15.6 \times 23.5 \mathrm{~mm})$ & FF $(35.9 \times 24 \mathrm{~mm})$ \\
\hline Resolution & $24 \mathrm{MP}(4000 \times 6000)$ & $42 \mathrm{MP}(7952 \times 5304)$ \\
\hline Sensor pixel & $15.28 \mu \mathrm{m}^{2}(3.9 \times 3.9 \mu \mathrm{m})$ & $20.43 \mu \mathrm{m}^{2}(4.5 \times 4.5 \mu \mathrm{m})$ \\
\hline Shutter & $\begin{array}{l}\text { Mechanical curtain }(\text { with rolling shutter } \\
\text { effect })\end{array}$ & $\begin{array}{l}\text { Mechanical central (without rolling } \\
\text { shutter effect })\end{array}$ \\
\hline Interchangeable lens & YES & NO \\
\hline Lens & Voigtlander Color-Skopar $21 \mathrm{~mm} \mathrm{F/4.0}$ & Carl Zeiss Sonnar T* 35mm F/2.0 \\
\hline Focusing system & mechanical & electronic \\
\hline Aperture setting & F/5.6 & F/4.0 \\
\hline Shutter setting & $1 / 1000 \mathrm{~s}$ & Auto $100-400$ \\
\hline ISO setting & Auto $100-800$ & $\mathrm{~s}$ \\
\hline
\end{tabular}

Table 2 Technical data of the cameras

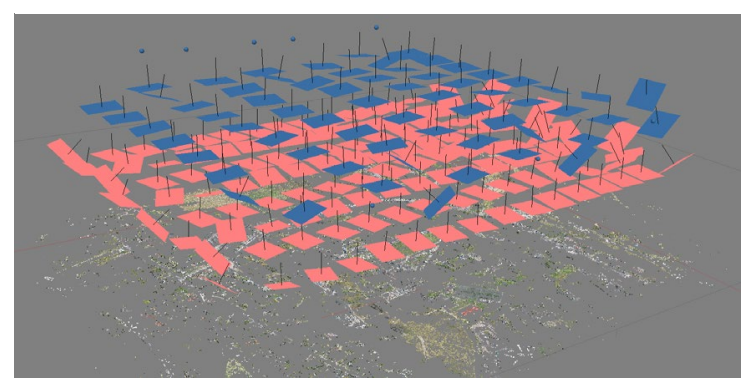

Figure 3 Cross flight configuration - variant 6 (Sony RX1RII)

Final flight configuration variants were as follow:

1. Mean GSD $2 \mathrm{~cm}$, overlap 60/60 \%, flight direction N-S

2. Mean GSD $2 \mathrm{~cm}$, overlap $60 / 60 \%$, flight direction W-E

3. Mean GSD $2 \mathrm{~cm}$, overlap $70 / 70 \%$, flight direction N-S

4. Mean GSD $3 \mathrm{~cm}$, overlap $60 / 60 \%$, flight direction W-E

5. Mean GSD $3 \mathrm{~cm}$, overlap $60 / 60 \%$, flight direction N-S

6. Cross flight: Variant $1+$ Variant 4

7. Cross flight: Variant $1+$ Variant 2

8. Cross flight: Variant $4+$ Variant 5

Due to different shutter, lens mount and focusing system of the cameras (Table 2), significant differences in calibration stability were expected. Sony a6000, due to the use of mechanical curtain shutter is affected by so called "rolling shutter effect", which in case of fixed wing UAV have to be compensated during bundle adjustment process (Vautherin, Rutishauser, 2016). It also has interchangeable lens system and consequently - greater instability of the principal point were expected. On the other hand, Sony RX1R II is a compact full-frame camera with mechanical central shutter and fixed lens.

To compare results of different georeferencing methods, UAV used were equipped with single-frequency, multiconstelation PPK receiver Emlid Reach M+. In order to obtain the most accurate time synchronization between camera and the receiver they were connected via camera hot shoe. The PPK receiver was used only for determining image coordinates and not for UAV navigation.

\section{CALCULATIONS}

Calculations were performed in variants depending on:

- Flight configuration

- Camera used

- Georeferencing method (PPK - with precise camera coordinates and without GCP, NAV/GCP - with approximate camera coordinates and $6 \mathrm{GCP}, \mathrm{PPK} / \mathrm{GCP}$ - with precise camera coordinates and $6 \mathrm{GCP}$ )

In georeferencing methods PPK and PPK/GCP camera precise coordinates were determined based on GNSS observation from PPK receiver. This calculation was made in open source RTKLib software in reference to local Base Station and regional CORS (36 km away from test field). In NAV/GCP variants approximated camera coordinates were obtained from standard code-based receiver.

Photogrammetry processing was performed with Agisoft Metashape Pro 1.5.3 and consisted of: 
1. Image alignment

2. Ground Control Points marking

3. Optimization (bundle adjustment)

4. Check Points (Group I) RMSE calculation

5. DEM and orthomosaicgeneration

It should be noted that to ensure the independence of calculations, image alignment process was made independently for each variant with High accuracy setting (pixel level matching). On the other hand, points marking on images were done only once for PPK 1-5 variants than they were copied for variants 6-8, NAV/GCP and PPK/GCP. This allowed to minimize the impact of marking inaccuracy between calculation variants. Depending on the calculation variant apriori accuracy settings were set up accordingly to values in Table 3 . It is worth to highlight that in PPK variants marker image measurements accuracy were set to 1000000 pixels in order to eliminate their influence on the adjustment.

\begin{tabular}{|c|c|c|c|}
\hline & PPK & $\begin{array}{c}\text { NAV } \\
\text { /GCP }\end{array}$ & PPK/ GCP \\
\hline Camera accuracy (m) & $0.02 / 0.08$ & 10 & $0.02 / 0.08$ \\
\hline Camera accuracy (deg) & 10 & 10 & 10 \\
\hline Marker accuracy (m) & $\mathrm{n} / \mathrm{a}$ & 0.02 & 0.02 \\
\hline Marker accuracy (pix) & 1000000 & 0.5 & 0.5 \\
\hline Tie point accuracy (pix) & 1 & 1 & 1 \\
\hline
\end{tabular}

Table 3 Apriori accuracy settings

For all variants cameras calibration parameters and Check Points RMSE (Group I) were calculated.

In addition, for variants 5 and 6 , after which the poorest and the best accuracies were expected, RMSE calculations were performed based on all control and check points (Group II and III). The points were measured based on generated orthomosaicss and DEM models. The measurements were made in QGIS software.

\section{RESULTS OF INVESTIGATION}

The first step of the investigation focused on determination of the final accuracy depending on the georeferencing method and camera used based on comparison of the RMSE values at the check points (Figure 5).

First of all, it should be noted that for all variants final 3D accuracies are below three times of the GSD and achieve $7.6 \mathrm{~cm}$ in the worst case scenario. That allows us to conclude that in all cases there are no models bending.

Regarding the georeferenced method we can recognize that in all PPK variants horizontal accuracy is lower than in other variants, which probably results from the inaccuracy of the coordinates of the cameras projection centres obtained from GNSS measurements. On the other hand, using the PPK method allowed to increase accuracy in vertical direction. This allows the statement that the use of precise image coordinates reduce correlation between camera calibration and images external orientation. And as was expected the highest accuracies were obtained in PPK/GCP variants.

In turn, when analysing the result according to the camera used, no significant differences were noticed.

Analysing the results according to the flight configuration we can conclude:

- As expected: cross flights configuration increase accuracy especially in vertical axis. The best results were obtained in variant 6 (with varying flight altitude)

- In this case flight direction has no significant influence on the result
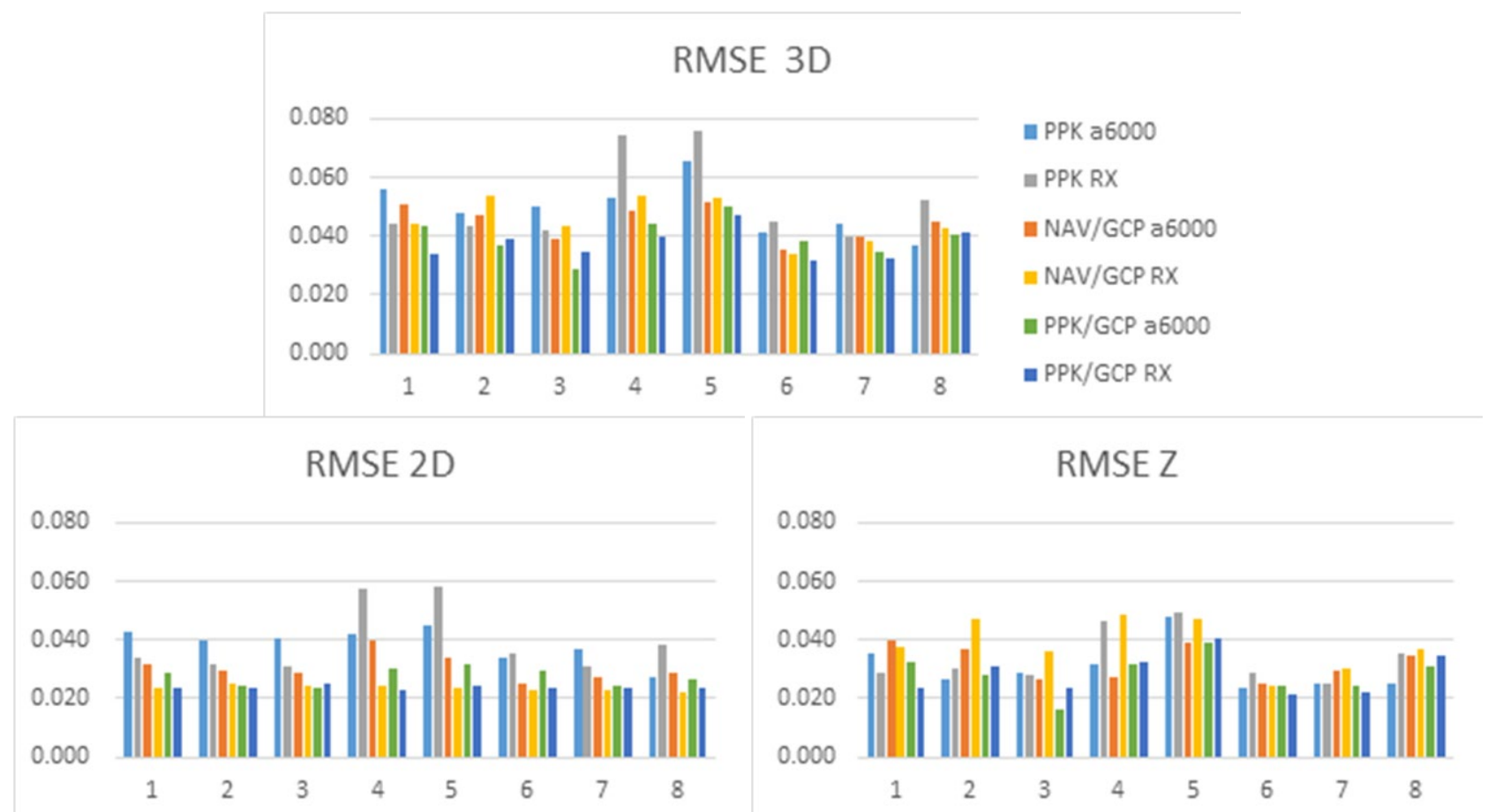

Figure 5 RMSE values at Check points (Group I) calculated in Agisoft 
- Increased overlap from $60 / 60 \%$ to $70 / 70 \%$ had positive influence on the results in variants with a6000 camera

- As expected: decreasing GSD from $2 \mathrm{~cm}$ to $3 \mathrm{~cm}$ results in accuracy decreasing

Second part of the investigation focused on determination of the cameras calibration stability. This kind of investigation was possible due to the fact that between each flight configuration there were no middle landings. It allowed to assume that there are no differences resulting from cameras constructions and insitu calibration accuracy.

Analysing the comparison of adjusted focal length and principal point values for Sony a6000 (Figure 6) and Sony RX1R II (Figure 7), it is clear that cameras differ significantly in terms of interior stability. It should be also noted that for both cameras variants PPK and PPK/GCP allowed to achieve greater stability of results than in NAV/GCP.
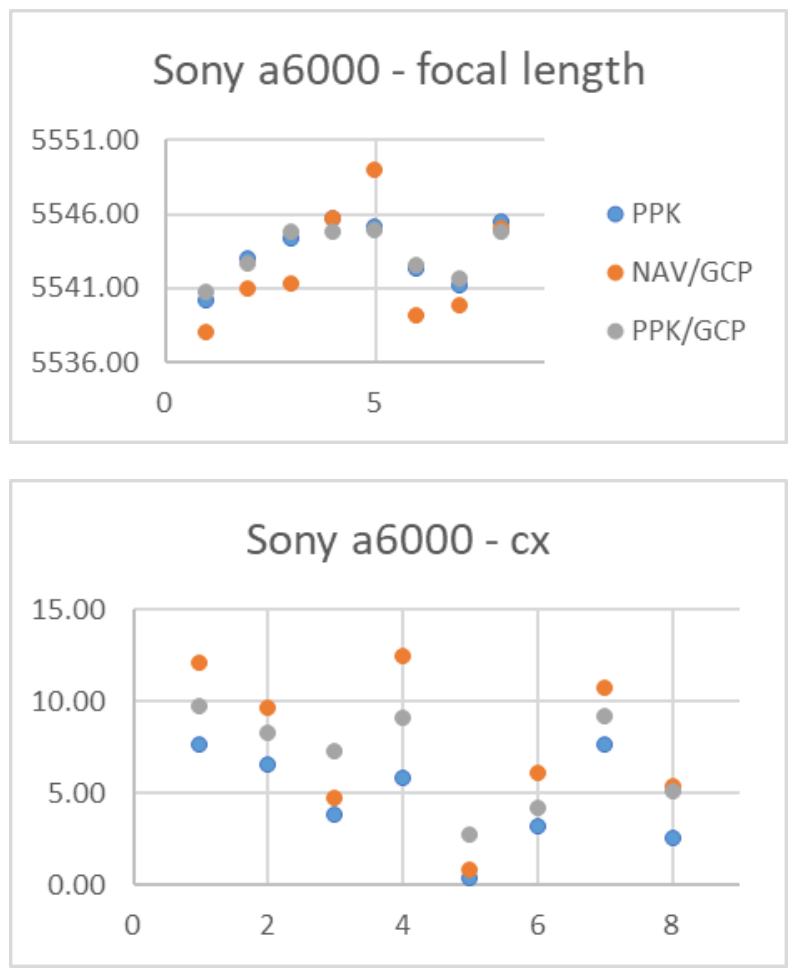

\section{Sony a6000 - cy}

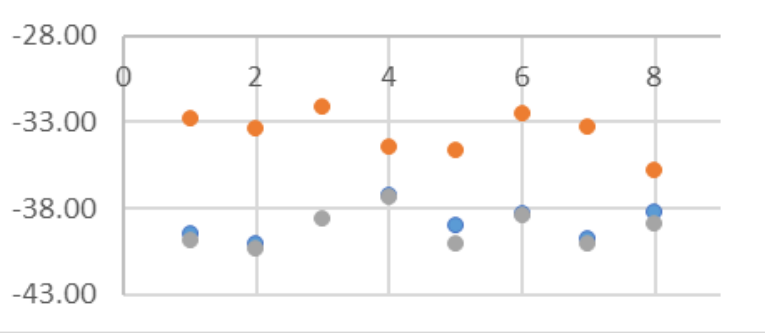

Figure 6 Sony a6000 in-situ calibration values
For Sony a6000 focal length value differs in 5 pixel range and it could be noted in correlation with flight altitude. But, in particular, attention should be paid to high variety of principal point position. Values in $\mathrm{X}$ axis direction are changing in almost 12 pixel range, which probably result from incomplete rolling shutter compensation.

At the same time, Sony RX1R II is characterized by a very high internal stability. Focal length and principal point position differ maximum by 3 pixels between variants. However, in the case of focal length, the difference between PPK and NAV variants is clearly visible. This probably results from insufficient georeference information in NAV variants.

In addition, charts of tie point's reprojection residuals (Figure $8,9)$ were analysed. It allowed to state that distortion model implemented in Agisoft is insufficient to eliminate distortion in Sony RX1R II camera. Despite the use of k1-k4 radial parameter and p1-p3 tangential on the residual chart there is still visible deviations with regular concentric distribution. This could significantly reduce final products accuracy.
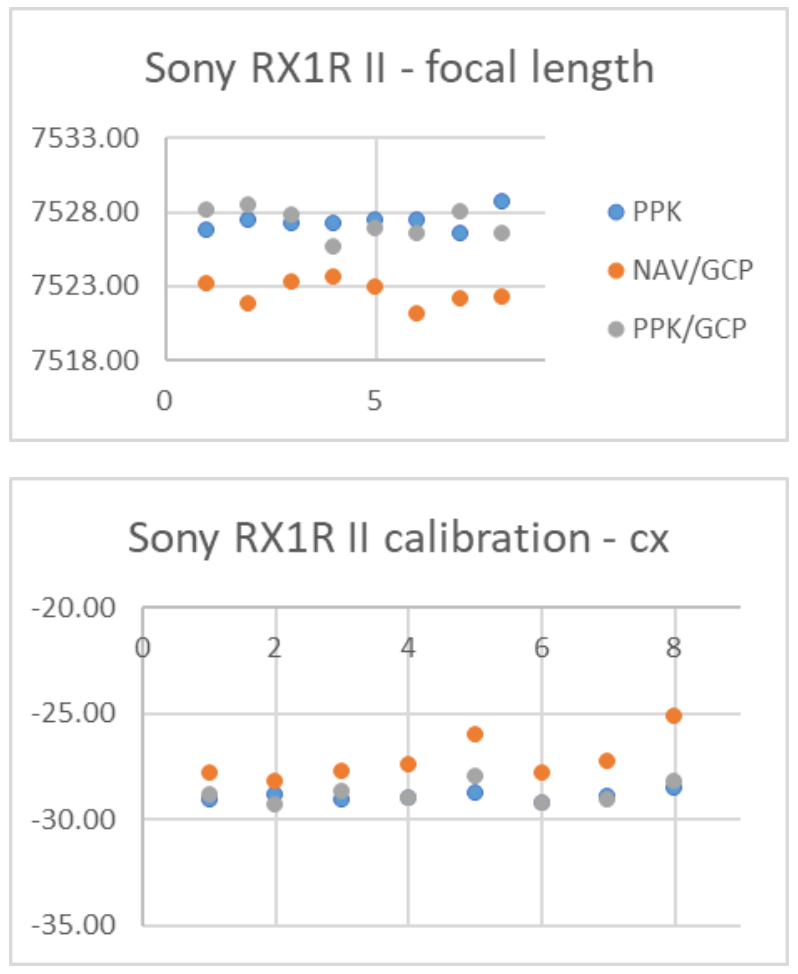

\section{Sony RX1R II calibration - cy}

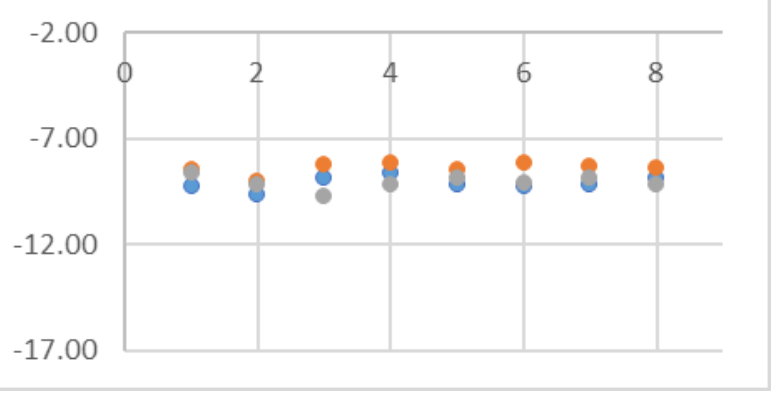

Figure 7 Sony RX1R II in-situ calibration values 


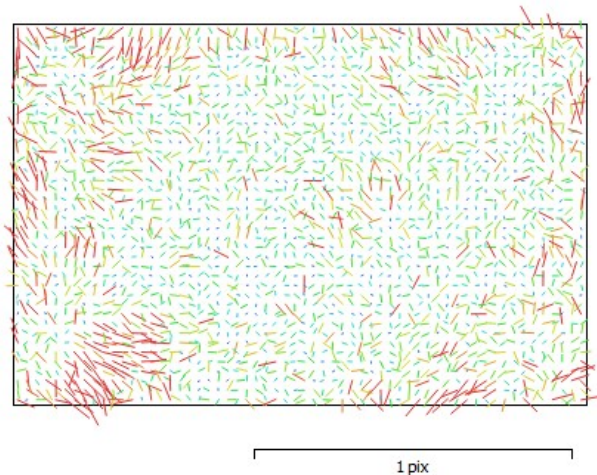

Figure 8 Sony a6000 reprojection residuals chart

On the contrary, in Sony a6000 case, model with k1-k3, p1-p2 and rolling shutter compensation allowed to eliminate most of the camera distortion errors.

The next stage of investigation was to compare RMSE values obtained in Agisoft (where 25 control points were measured directly on images) to values obtained in QGIS, where almost 150 control points (depending on variant) were measured. The comparison was done for variants 5 and 6 which were identified as the poorest and the best one.

The result (Figure 10) shows that there is no significant difference between values. However, values obtained from QGIS measurement are in most cases slightly higher than those from Agisoft..

\section{CONCLUSIONS}

To sum up, the prepared test field allowed for investigation of accuracy of UAV-based surveying.

The possibility of obtaining centimetre-level accuracy was confirmed for both the traditional method employing Ground Control Points as well as for direct georeference method involving precise cameras coordinates. As it was expected based on previous researches (Gerke, Przybilla 2016), the highest accuracies were obtained in variants including cross flight pattern and varying flight altitude.

Despite significant differences in the internal stability of both cameras, values of the obtained RMSE errors were at a similar level, which indicates a large degree of independence in the

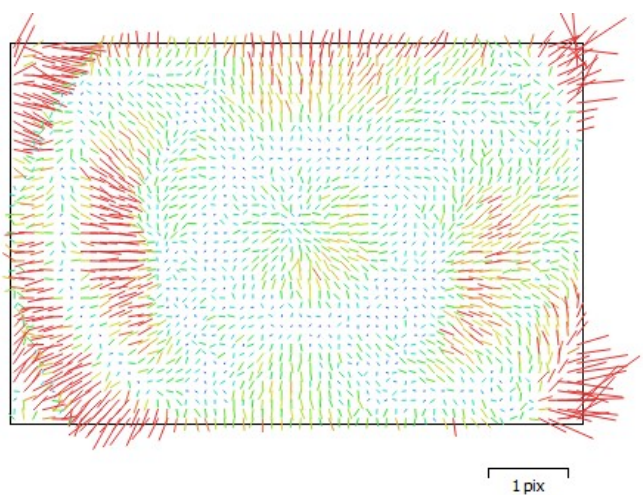

Figure 9 Sony RX1R II reprojection residuals chart

selection of coefficients in the case of the in-situ calibration method.

The use of the PPK receiver increases the consistency of the obtained calibration factors.

As part of further research on the test field, it is planned to perform analyses of the resistance of the photo block to the occurrence of outliers. Consequently, an experiment with different GCP and CP location, also on the roofs would be relevant.

\section{ACKNOWLEDGEMENTS}

This research was conducted using funds from Ministry of Science and Higher Education Research Grant No. DI 2016004346 and the AGH University of Science and Technology Research Grant No. 11.11.150.949.

\section{REFERENCES}

Cramer, M., Bovet, S., Gültlinger, M., Honkavaara, E., McGill, A., Rijsdijk, M., Tabor, M., Tournadre., V., 2013: On the use of RPAS in national mapping - the EuroSDR point of view.

ISPRS - Int. Arch. Photogramm. Remote Sens. Spatial Inform. Sci., XL-1/W2, 93-99.

Cramer, M., Przybilla, H.-J., Zurhorst, A., 2017: UAV Cameras: Overview and Geometric Calibration Benchmark, ISPRS - Int. Arch. Photogramm. Remote Sens. Spatial Inform. Sci., Vol. XLII-2/W6, 85-92.

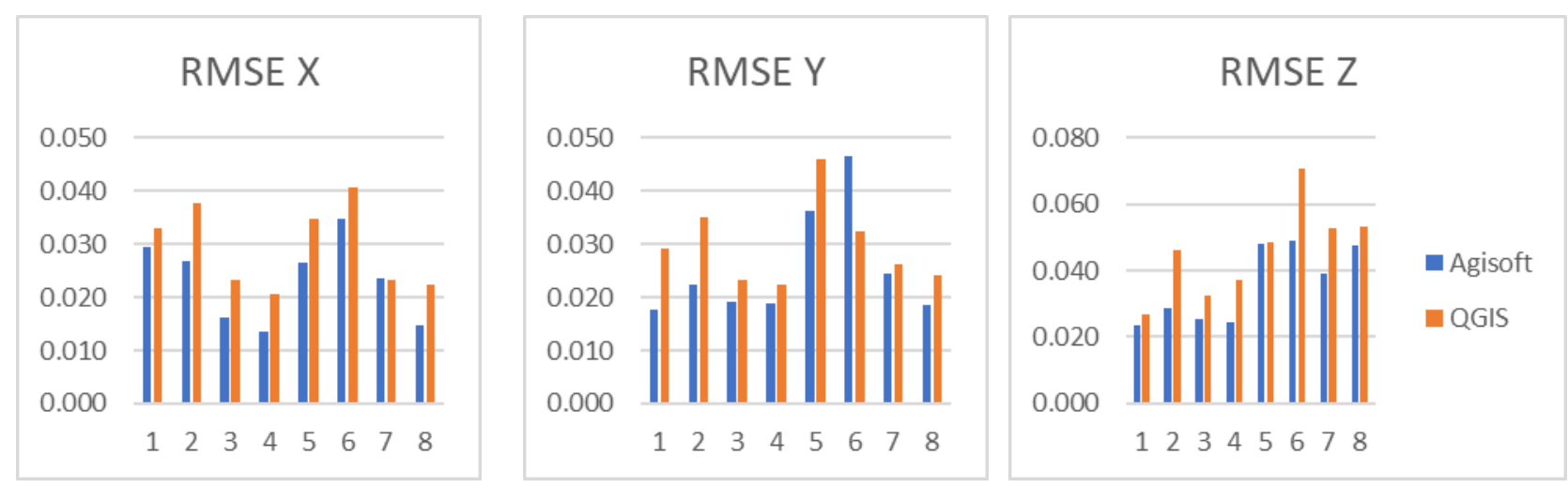

Figure 10 RMSE values from Agisoft and QGIS 1-Variant 6 PPK a6000, 2-Variant 6 PPK RX, 3-Variant 6 NAV/GCP a6000, 4Variant 6 NAV/GCP RX, 5-Variant 5 PPK a6000, 6-Variant 5 PPK RX, 7-Variant 5 NAV/GCP a6000, 8-Variant 5 NAV/GCP RX 
Daakir, M., Zhou, Y., Pierrot Deseilligny, M., Thom, C., Rupnik, E., 2019: Improvement of photogrammetric accuracy by modeling and correcting the thermal effect on camera calibration. ISPRS Journal Of Photogrammetry And Remote Sensing, vol. 148, 142-155. https://doi.org/10.1016/j.isprsjprs.2018.12.012

Gerke, M. and Przybilla, H.-J., 2016: Accuracy Analysis of Photogrammetric UAV Image Blocks: Influence of Onboard RTK-GNSS and Cross Flight Patterns. Photogrammetrie Fernerkundung- Geoinformation (PFG), 2016 (1), 17-30. DOI: $10.1127 / \mathrm{pfg} / 2016 / 0284$

James, M.-R., Robson, S, d'Oleire-Oltmanns, S., Niethammer, U., 2017: Optimising UAV topographic surveys processed with structure-from-motion: ground control quality, quantity and bundle adjustment. Geomorphology, vol. 280, 51-66. https://doi.org/10.1016/j.geomorph.2016.11.021

Luhmann, T., Fraser, C., Maas, H.-G., 2015: sensor modelling and camera calibration for close-range photogrammetry. ISPRS Journal Of Photogrammetry And Remote Sensing. VOL. 115, 37-46. https://doi.org/10.1016/j.isprsjprs.2015.10.006

Kurczynski, Z., Bakuła, K., Karabin, M., Kowalczyk, M., Markiewicz, J.S., Ostrowski, W., Podlasiak, P., Zawieska, D., 2016: The possibility of using images obtained from the UAS in cadastral works. Int. Arch. Photogramm. Remote Sens. Spatial Inf. Sci., XLI-B1, 909-915. https://doi.org/10.5194/isprsarchives-XLI-B1-909-2016

Kedzierski, M., Fryskowska, A., Wierzbicki, D., Nerc, P., 2016: Chosen aspects of the production of the basic map using UAV imagery. Int. Arch. Photogramm. Remote Sens. Spatial Inf. Sci., XLI-B1, 873-877. https://doi.org/10.5194/isprs-archives-XLIB1-873-2016

Nex, F., Remondino, F., 2014: UAV for 3D mapping applications: a review. F. Appl Geomat 6:1, 1-15. https://doi.org/10.1007/s12518-013-0120-x

Sanz-Ablanedo, E.; Chandler, J.H., Rodríguez-Pérez, J.R.; Ordóñez, C., 2018: Accuracy of Unmanned Aerial Vehicle (UAV) and SfM Photogrammetry Survey as a Function of the Number and Location of Ground Control Points Used. Remote Sens., 2018, 10, 1606.

Stöcker, C., Nex, F., Koeva, M., Gerke M., 2019: UAV-Based Cadastral Mapping: an Assessment of the Impact of Flight Parameters and Ground Truth Measurements on the Absolute Accuracy of Derived Orthoimages. ISPRS - Int. Arch. Photogramm. Remote Sens. Spatial Inform. Sci., Vol. XLII2/W13, 613-617. https://doi.org/10.5194/isprs-archives-XLII-2W13-613-2019

van Hinsberg, W., Rijsdijk, M., Witteveen, W.,2015: UAS for cadastral applications: testing suitability for boundary identification in urban areas. GIM Int., 27, 20-25.

Vautherin, J., Rutishauser, S., Schneider-Zapp, K., Choi, H. F., Chovancova, V., Glass, A., Strecha, C., 2016: Photogrammetric Accuracy and Modeling of Rolling Shutter Cameras. ISPRS Annals of Photogrammetry, Remote Sensing \& Spatial Information Sciences, 3(3), 139-146. doi:10.5194/isprsannalsIII-3-139-2016 